\title{
Design of Multi-channel MAC Mechanism for WIA-PA
}

\author{
Jianming $\mathrm{Liu}^{1}$, Lili $\mathrm{Xu}^{2}$ \\ ${ }^{1}$ Computer Department, Weifang Medical University, Weifang, 261053, China \\ 2 Joint first authors,W eifang Vocational College,Weifang, 261042, China
}

Keywords: WIA-PA; Multi-channel; MAC mechanism; Channel Assignment Algorithm

\begin{abstract}
WIA-PA is an industrial wireless networking protocol standard used in process automation. One mechanism based on the WIA-PA multi-channel MAC has been proposed to solve industry wireless sensing questions in network channel disturbance and medium turning on conflict. The mechanism mainly includes the multi-channel MAC mechanism design proposal and the channel assignment algorithm design about WIA-PA.
\end{abstract}

\section{Introduction}

WIA-PA is an industrial wireless networking protocol standard used in process automation. This protocol introduces the wireless communication technology into the field of industrial process control, and its network communication has the properties of real-time communication, high reliability, and low power consumption [1].

In this paper, one mechanism based on the WIA-PA multi-channel MAC has been proposed to solve industry wireless sensing questions in network channel disturbance and medium turning on conflict, and enhance the network volume of goods handled and timeliness. This article introduces the multi-channel MAC mechanism design proposal and the channel assignment algorithm design about WIA-PA. .

\section{Design Proposal}

i) In the WIA-PA network system, it must reasonably use the physical level resources; effectively avoid the universal existence conflict and the disturbance question of the single channel correspondence mechanism. The multi-channel MAC mechanism may avoid the communication jamming and the conflict in the full use channel resources foundation [2].

ii) At present, the multi-channel MAC mechanism which exists has respectively good and bad points. It can not apply to WIA-PA directly, but at present there isn't the multi-channel MAC plans Suitable for WIA-PA.

iii) WIA-PA has mainly been used in the industry control domain, in which the working conditions is complex. The specific frequency band channel quality is easy to receive the disturbance. Once the traditional channel assignment algorithm has been used, the channel assigns is difficult to change. It cannot promptly redistribute the channel for the node which has been disturbed. It affects the correspondence timeliness.

iv) Nearby the SINK node, the data transmission capacity is big. It is easy to create the transmission conflict. Under the CAMA/CA mechanism, the SINK node as well as a bunch of first node must carry on the channel cut unceasingly, and increase network consumption.

In view of the above considerations, it is necessary for multi-channel MAC mechanism based on WIA-PA. It consists of two main areas of content: static and dynamic channel allocation algorithm design, and design of communication mechanisms within the cluster .

\section{Channel Assignment Algorithm}

A combination of static and dynamic channel allocation algorithms in Channel allocation is used. Static channel allocation is mainly used for fixed channel assignment, and mainly in the cluster network initialization process. It can improve Implicit-Consensus algorithm in MMSN network. It 
uses a single node in the cluster in place of the algorithm, ensures a skip next cluster use different channel, and prevents adjacent interference problems. Dynamic channel allocation is mainly used for cluster and node communication channel of distribution in a disturbed family. In this algorithm, it is used to rate predictions of the channel quality assessment indicators, when the forecast receiving a packet is lower. It indicates that the channel is severely disrupted, and it will reassign channel for the cluster head node within the Group that has been interfered.

\section{Static Channel Allocation Algorithms}

Static channel allocations are mainly based on channel allocation for network initialization process. After the W1A-PA cluster partition, it has a certain distance between clusters of subject. In order to avoid disturbance between adjacent inter-cluster communications, it is only guaranteed among neighboring clusters using a variety of communication channels in the channel distribution network initialization process.

The Implicit-Consensus algorithms is used for channel allocation between clusters, its specific scheme is as follows:

Input: Node a's ID number, Node a's neighbors `ID number within two communication hops[3].

output: the assigned frequency number for node a .

int index $=0$;

int Frequency Number= -1;

if

FOUND = false;

break;

end if

end for

if FOUND then

Frequency Number = index;

else

index ++ ;

end if

until Frequency Number $>-1$;

Based on the Implicit-Consensus [4], it uses a single cluster instead of node for limiting the amount of channel demand, not using a random-number generator. A direct comparison of the cluster head node ID numbers determines channel competition results. The cluster head node maintains two data structures: o-cluster cluster ID number and a list of available channels. The main idea is as follows:

FrequmcyNum $_{n}=$ Frequmcy Re source
$I D_{n}=\min \left\{I D_{1}, I D_{2}, I D_{3}, I D_{4}, I D_{5} \ldots \ldots\right\}$

FrequmcyNum $_{n}$ is a serial number of channels that are assigned after cluster $\mathrm{n}$ competition communication; FrequmcyResource $_{n}$ is a serial number of channels to be distributed in the FrequmcyNum $_{n}$ for this round of the competition.

Algorithm implementation steps are as follows:

i) The various network parameters including the ID number of cluster head and channel serial number are initialized, the channel list ascend by the serial number.

ii) Cluster head receives a jump of cluster head the broadcast information, and removes adjacent cluster head of the clusters,.

iii) The result of the competition is judged, judge standards is described in formula (1). If the channel competition of cluster head is successful, it will exit the channel competition. If the channel competition fails, it will go on the competition for a new round of channel.

The flow chart of fixed channel allocation is shown as follows. 


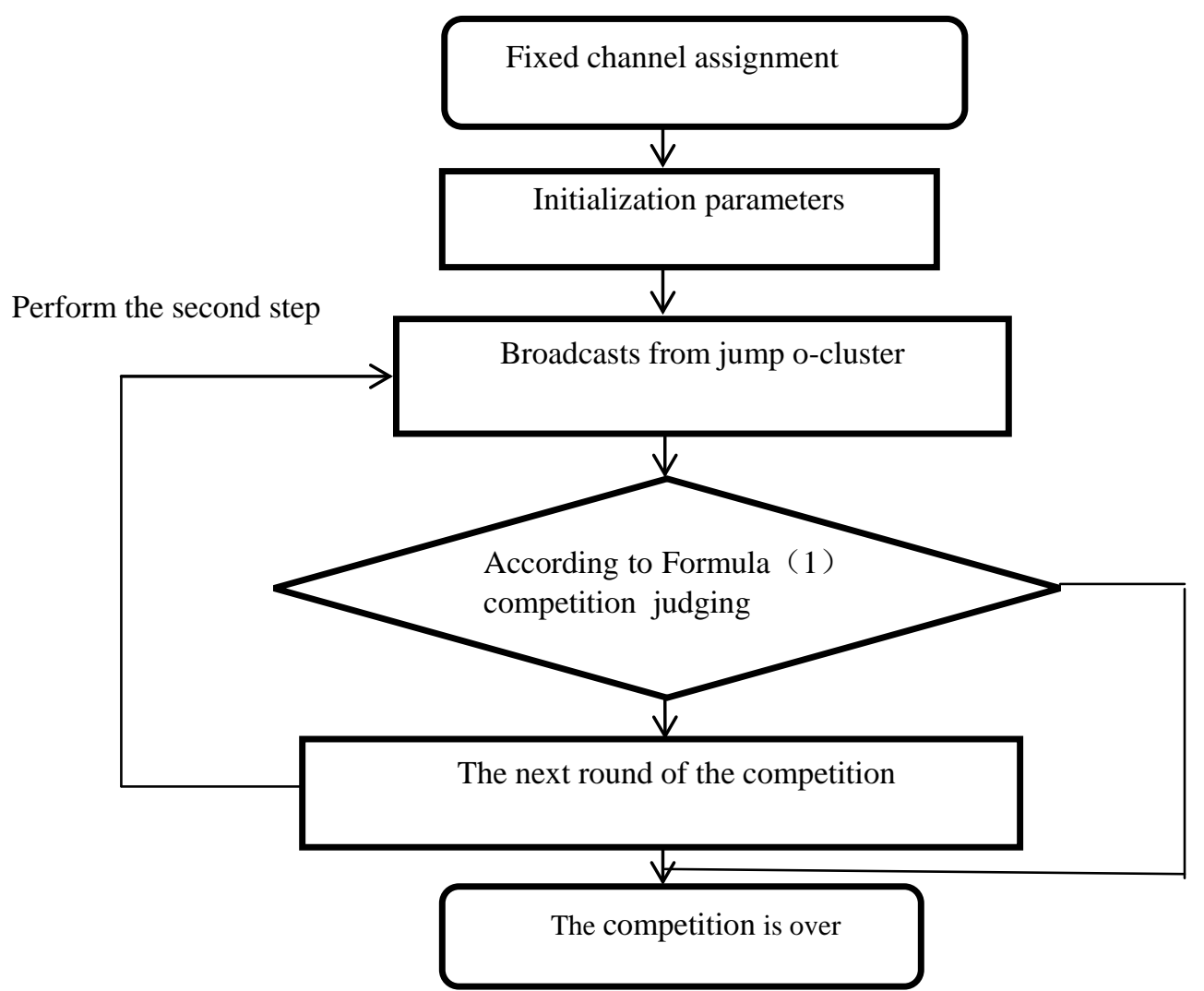

Fig1.The flow chart of fixed channel allocation

\section{Dynamic Channel Allocation Algorithms}

At the time of dynamic channel allocation, using weighted moving average method, it is able to effectively evaluate the channel quality; this method can effectively reduce errors. Package rate forecasts can better reflect the actual quality of the communication channel, if the forecast is too low. This indicates that the communications channel has become poor, and been disturbed. Cluster head node interference should be assigned a communication channel.

In this article, according to a period of time to meet forecasts and package rate Current package rate measurements, the algorithm of EWMA is used to calculate channel meeting package rate forecast of the next moment, the algorithm is as follows:

$$
Q_{t}\left(n, F_{i}\right)=\frac{m_{t}\left(n, F_{i}\right)}{M_{t}\left(n, F_{i}\right)}
$$

$E_{t}\left(n, F_{i}\right)=\theta E_{t-1}\left(n, F_{i}\right)+(1-\theta) Q_{t}\left(n, F_{i}\right)(0<\theta<1)$

$Q_{t}\left(n, F_{i}\right)$ is the meeting package rate statistics of node $\mathrm{n}$ on channel $F_{i}$;

$m_{t}\left(n, F_{i}\right)$ is the number of packages from node $n$ in $t$ time;

$M_{t}\left(n, F_{i}\right)$ is the packets that node $n$ gives to cluster head on the channel $F_{i}$ in $t$ time;

$E_{t}\left(n, F_{i}\right)$ is the current forecast of meeting package rate;

$E_{t-1}\left(n, F_{i}\right)$ is the a moment before forecast of meeting package rate;

The Flow chart of Dynamic Channel Allocation is shown as follows:

\section{Communication Design of Multi-channel MAC Protocol}

WIA-PA network communication cycle can be divided into two stages: communication between cluster and internal communication. In this paper, communication between the virtual link mechanism is used based on time division multiplexing, to complete the data transmission between clusters. Within the cluster communication stage, keating Polling communication mechanism is 
used to complete the scene node data collection. Multi-channel MAC protocol of the communication process is shown as follows.

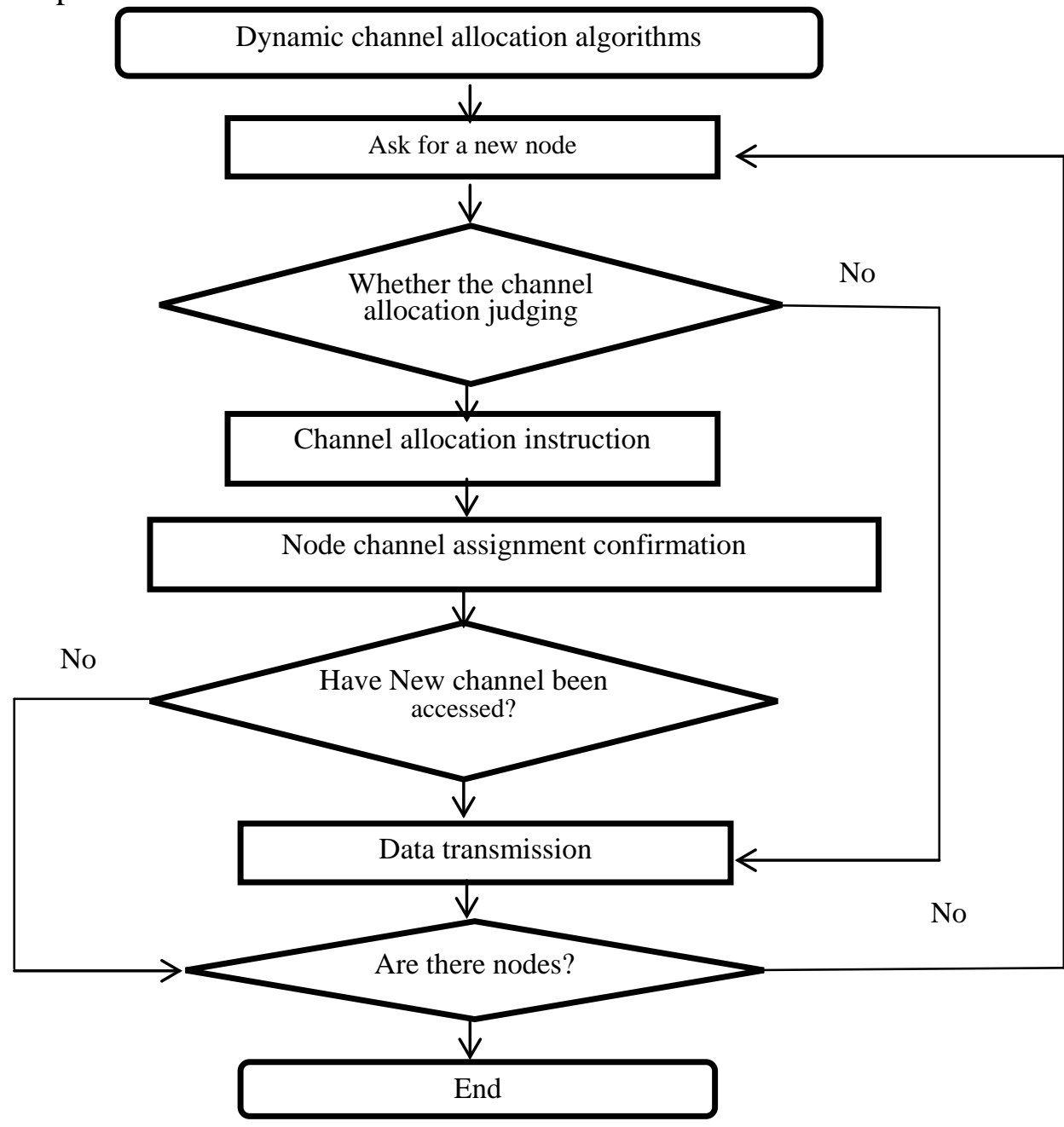

Fig2: Flow chart of Dynamic Channel Allocation

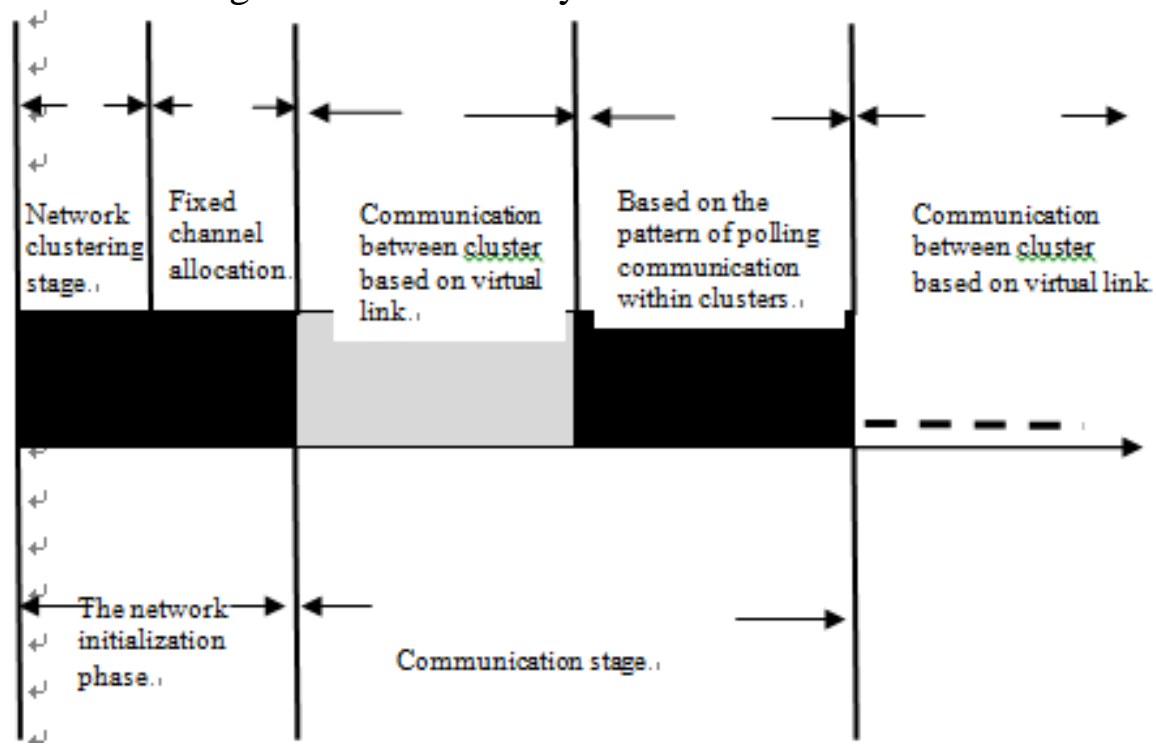

Fig3:Flow chart of communication process on WIA-PA Multi-channel MAC Protocol

\section{Design of WIA-PA multi channel MAC communication mechanism between clusters}

In communication between clusters in the design process, through the use of virtual link mechanism, it can be combined with Multi-Radio approach, and transmit data to the SINK node. After the network initialization is completed, each cluster will assign an ID number in the capital, and follow the channel assignment algorithm described previously, channel allocation, 
communication channel so that each cluster will have a base in the capital. The regulation of network nodes (SINK) as the Center, forms thousands of link (virtual link), and each link communication channel selection is directly connected with the SINK node cluster used by the base channel. For inter-cluster data exchange, cluster at the same link communication channel will own the channel used by the switch to a common link through the TDMA mode, and data is transmitted to the cluster head connected with the sink node. Then cluster head transmits the data to SINK nodes in order. Schematic diagram of virtual communication between clusters link is shown as follows.

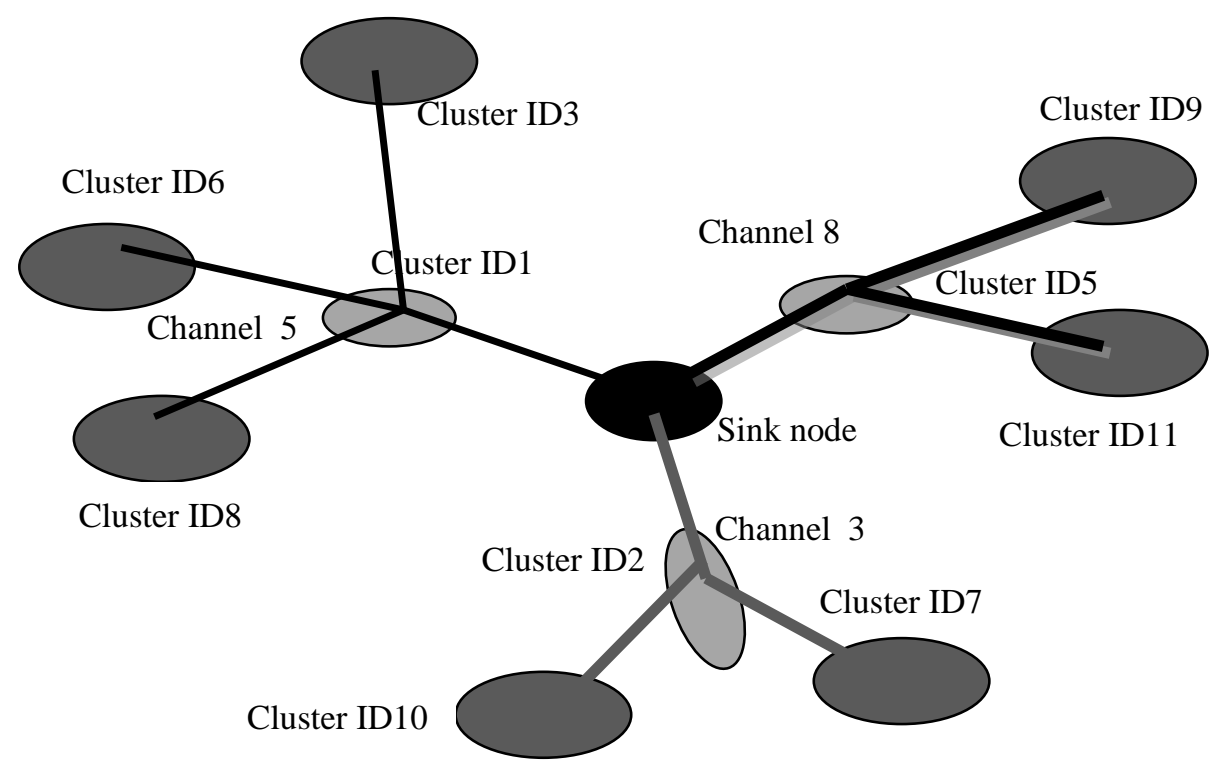

Fig4. Schematic diagram of virtual communication between clusters

\section{Design of WIA-PA multi channel MAC communication mechanism in clusters}

In clusters within communications, round polling mechanism is used. The same channel of node is arranged together to stay polling. Internal base channel node is priority to exchange data. Cluster between control channel of node is last to exchange data. The other channel is arranged by node number descending [6-8].

The basic principles of Based on polling the intra cluster communication are as follows:

i) It is aligned with all of the same channel using network nodes. communication resource is allocated by the routing node.

ii)All the nodes using based channel are priority to allocate communication resource.

iii) In addition to the cluster-channel basis, the allocation of other channels of communication slots is ordered by descending.

iv) The nodes using the cluster channel is arranged the final surface of the queue. These nodes allocate communication resource after other nodes has completed communicate, and complete data exchange.

\section{Conclusion}

The paper has proposed WIA-PA based on multi-channel MAC Protocol for wireless sensor networks. Compared to the traditional single-channel MAC, in the case of full use of channel resources, it can reduce transmission conflicts, and increase network throughput. It is perfect for WIA-PA industrial wireless networks in reliable transmission and real-time requirements, but it is also needed to improve further. 


\section{Acknowledgements}

The research work was supported by the Shandong Province Medical Science and Technology Program under Grant No. 2014WS0460.

\section{References}

[1]Bo Yu, Jianzhong Li, Yingshu Li. Distributed Data Aggregation Scheduling in Wireless SensorNetworks[C].IEEE INFOCOM 2009, 2009:373-381.

[2]ZhengT, Radha krishnans. SaranganV. PMAC: an adaptive energy-efficient MAC Protocol for wireless senor networks[C].In Proeeedings of the 19th IEEE International Parallel and Distributed Processing Symposium. New York, USA. 2011:66-72.

[3]Akyildiz IF, Wang X, Wang W. Wireless mesh networks: a survey [J]. Computer netwoks,2013,47(4):445-487.

[4]A.Ei-Hoiydi, J.D.Decotignie.Low Power MAC Protocols for Infrastructure WSN[C].Swiss Center for Electronics and Microtechnology, 2012.

[5]Yih W, Goodman J, Vanderwende L, etal. Multi-document summarization by maximizing informative content-words[C]//Proceedings of he 20th International Joint Conference on Artificial Intelligence (IJCAI-07),2010: 1776-1782.

[6] [Awadhesh Kumar,Neeraj Tyagi. Cross-layer self organized routing protocol for MANET[A]. Proceedings of International Conference on Computer Communication and Management (ICCCM 2011) [C]. 2011

[7] An Open Architecture for the Routing Protocols Design in Ad hoc Networks[A]. Proceedings of 2010 3rd IEEE International Conference on Computer Science and Information Technology VOL.7[C]. 2010.

[8] SHEN Bin,LIU Xin-lei,QIN Xian-li,FU Ming-chao. Research of Wireless routing protocol Used in Underground Wireless Gas Monitoring Network Based on Star-bus Topology and non Protocol Stack[A]. Proceedings of the 3nd International Conference on Digital Manufacturing \& Automation(ICDMA2012)[C]. 2012. 